\title{
Kernos
}

Revue internationale et pluridisciplinaire de religion grecque antique

$24 \mid 2011$

Varia

Miriam VALDÉS GUÍA, El nacimiento de la autoctonía ateniense : cultos, mitos cívicos y sociedad de la Atenas del s. VI a. C.

\section{Claudine Leduc}

\section{(2) OpenEdition}

\section{Journals}

Édition électronique

URL : http://journals.openedition.org/kernos/1945

DOI : 10.4000/kernos. 1945

ISSN : 2034-7871

Éditeur

Centre international d'étude de la religion grecque antique

Édition imprimée

Date de publication : 1 janvier 2011

Pagination : 325-328

ISSN : 0776-3824

Référence électronique

Claudine Leduc, « Miriam valdés guía, El nacimiento de la autoctonía ateniense : cultos, mitos cívicos y sociedad de la Atenas del s. VI a. C. », Kernos [En ligne], 24 | 2011, mis en ligne le 14 octobre 2011, consulté le 21 septembre 2020. URL : http://journals.openedition.org/kernos/1945 ; DOI : https:// doi.org/10.4000/kernos. 1945 
Kernos 24 (2011), p. 325-368.

\title{
Revue des Livres
}

\section{Comptes rendus et notices bibliographiques}

\author{
Miriam VALDÉS GUÍA, El nacimiento de la autoctonía ateniense : cultos, mitos cívicos y \\ sociedad de la Atenas del s. VI a. C., Madrid, Publicaciones Universidad Com- \\ plutense de Madrid, 2008. 1 vol. $17 \times 24 \mathrm{~cm}, 274$ p. ('Ilu. Revista de Ciencias de las \\ Religiones, 22). ISBN : 978-84-669-3063-5.
}

L'A. se réfère d'entrée aux travaux de Nicole Loraux (p. 7) qui ont mis en évidence tous les «bénéfices » que les Athéniens tiraient, au temps de la démocratie (Ve-IVe s.), de leur façon de se raconter, et de raconter aux autres Grecs la merveilleuse naissance du fondateur de leur cité. Le corps civique est conçu comme un genos, un groupe gentilice exclusivement masculin, dont Érichthonios, engendré par Héphaïstos, porté par Gè et élevé par Athéna, est le père fondateur et Athéna, comme l'a voulu son fils adoptif, l'éponyme. Tous les descendants du premier Né de la Terre (Seuil, 1996) se transmettent de père en fils son autochtonie et doivent à sa paternité fondatrice de former une fraternité. Tous égaux et tous bien-nés, ils ont entre eux des relations de philia et peuvent, vis-à-vis des étrangers, tirer gloire collectivement d'une aussi noble et aussi ancienne origine. Pour N.L. le mythe développe, dans le langage qui est le sien, les thèmes de l'idéologie démocratique qui tait les divisions de la polis, exalte sa cohésion et glorifie l'archê qu'elle exerce dans le monde grec.

Pour M.V.G. cette version des origines de la cité, qui fait son apparition dans l'iconographie vers 500, est déjà en place lors de l'adoption de la politeia de Clisthène. Ce «mythe politique », dit-elle, est façonné - sinon construit - à partir de données plus anciennes, au VIe s. (p. 7). Dès la promulgation de la politeia de Solon, il fait partie de l'idéologie de la cité (p. 8) « dont il cimente en même temps qu’il reflète les acquisitions sociales et politiques, c'est-à-dire la liberté » qui est non seulement la libération de la douleia mais la participation à la vie politique, auparavant fondée «sur la naissance et le lignage ». Ces dernières années, les débats sur les origines de la démocratie athénienne ont refleuri en liaison notamment avec l'hommage à M.H. Hansen, les réunions en l'honneur du 2500e anniversaire de la politeia de Clisthène et le colloque Solon of Athens. M.V.G. se rallie donc à ceux qui soutenaient, comme R.W. Wallace - et contrairement à M.H. Hansen, J. Ober, K.A. Raaflaub et bien d'autres - que la démocratie était déjà dans la politeia de Solon.

Son projet, qui replace l'élaboration du mythe d'Érichthonios et des origines de la cité dans son contexte historique, est séduisant dans la mesure où il entend transgresser les catégories realia/fictions, histoire/anthropologie de l'imaginaire, religion/politique, diachronie/synchronie - où la recherche apprécie souvent de se couler. Il est aussi très ambitieux. Le choix de ces voies traversières exige un énorme travail de recherche car il impose de croiser des sources et des travaux de factures très différentes. En témoigne l'ampleur de la bibliographie de M.V.G., notamment en ce qui concerne les ouvrages en langue anglaise! Mais surtout le croisement fiction/ realia repose, lorsqu'il s'agit du VI s., sur des «sables mouvants », comme le dit F. Ruzé, des sables si mouvants que, quelle que soit la question examinée, l'absence de tout consensus contraint à la reprise de l'ensemble du dossier. Or j'ai l'impression que, si l'A. s'engage person- 
nellement lorsqu'il s'agit de quêter les indices qui lui permettent de retrouver dans les rituels du $\mathrm{VI}^{\mathrm{e}} \mathrm{s}$. la place des divinités qui sont impliquées dans la naissance d'Érichthonios, elle s'en tient à la doxa lorsqu'il s'agit des réalisations attribuées à Solon ou à Pisistrate. Plusieurs termes, ne serait-ce que celui de dèmos, auraient dû relever, me semble-t-il, d'une étude sémantique.

M.V.G. précise l'entrée de sa recherche. Il s'agit d'étudier la réorganisation, au cours du VIe s., de la tradition homérique sur la naissance autochtone du premier roi des Athéniens: dans l'Odyssée, il s'agit d'Érechthée, un fils de la terre, protégé d'Athéna qui partage sa vaste demeure; au cours du VI e s., Érechthée est évincé par un autre protégé d'Athéna - dont il devient le petit-fils dans la généalogie royale - Érichthonios qui, lui aussi, est né de la terre mais a été engendré par Héphaïstos. Ce remaniement de la tradition doit être mis en rapport, ditelle, avec l'intégration de la terre libérée par Solon dans un processus de développement de la propriété paysanne, de revalorisation de l'artisanat, surtout à partir de la tyrannie de Pisistrate, et d'incorporation du dèmos dans la politeia. Cette extension de la citoyenneté, ajoute l'A., suppose, comme le pense Ian Morris, un transfert des valeurs qui étaient celles de l'aristocratie (la détention de la terre, la caution des ancêtres, la noblesse de la naissance, la conduite héroïque à la guerre) vers le dèmos et cette nouvelle idéologie englobe tout le corps civique par delà ses deux grands clivages, sa partition fonctionnelle (Eupatrides, géomores et démiurges) et sa partition censitaire (Pentacosiomédimnes, chevaliers, zeugites et thètes) (p. 9). C'est alors que M.V.G. élargit la problématique qu'elle vient d'annoncer (p. 10) : elle entend consacrer son ouvrage à une analyse de l'idéologie du dèmos en portant une attention spéciale à ses aspects religieux et culturels, sans renoncer pour autant à celle de tout le corps civique, un corps civique qui inclut les aristocrates et, ce qui est plus curieux, les femmes. Pourquoi ce glissement? Est-ce au nom de la formation d'une identité collective qui engloberait désormais tout le groupe social ? Serait-ce une « commodité » pour inclure deux chapitres qui font preuve d'une certaine indépendance vis-à-vis du thème annoncé ? Sur les six chapitres de l'ouvrage, quatre s'essayent à démontrer que la version du mythe de l'autochtonie en usage dans la cité démocratique est en place dès le VI $\mathrm{s}$. Chap. I : Un nouvel ancêtre pour les Athéniens: Apollon Patroos. Ion et la participation du dèmos à la politeia. Chap. II : Gè, la Terre d'Athènes, et les Genésia : la paysannerie de l'Attique. Chap. III : L'introduction d'Héphaïstos dans l'imaginaire athénien et la promotion des artisans. Chap. IV : La naissance d'Érichthonios et les Panathénées: la création d'un mythe. Les deux derniers chapitres en revanche sont assez éloignés d'une telle préoccupation; le chap. V: Athéna contre Aphrodite : les femmes et la citoyenneté, revisite les propositions d'un travail précédent; le chap. VI : Les dieux libérateurs : de Zeus Eleutherios à Dionysos Eleuthereus, répond aux suggestions d'un article de K.A. Raaflaub.

Comme l'indiquent les intitulés des quatre premiers chapitres, M.V.G. reproduit, de l'un à l'autre, le même modèle de démonstration. Les acteurs divins qui, au Ve-IVe s., interviennent dans la naissance d'Érichthonios sont étudiés au cas par cas.

$$
\begin{gathered}
\text { Héphaïstos } \sim \text { Gè } \sim \text { Athéna } \\
\text { Érichthonios } \\
\text { (Pandion) } \\
\text { Érechthée } \\
\text { Xouthos + Créuse }+ \text { Apollon (Pythien) } \\
\text { Ion }
\end{gathered}
$$

L'A. commence par rassembler, avec une pointilleuse érudition, tous les indices qui attestent leur présence dans les rituels dès les grands changements du VIe s. et laissent deviner des configurations susceptibles de préfigurer leurs rôles dans la naissance d'Érichthonios. L'état des sources ne facilite pas toujours cette quête et le souci de tout prouver nuit parfois à la démonstration. Un exemple (chap. I) : le culte d'Apollon Patroos est attesté (temple de l'Agora 
dite de Solon) sous les Pisistratides et il serait en relation avec Delphes (p. 16). Faut-il en conclure pour autant qu'Apollon Patroos et Apollon Pythien ne font qu'un? Son culte se substitue alors à celui de Zeus Patroos (p. 15). Mais, en fait, le dieu est depuis longtemps présent sur le territoire. À partir d'une unique occurrence - un fragment de Philochore M.V.G. se croit autorisée à l'assimiler à Hélios (p. 21). Honoré avec Athéna et Poséidon, cofondateurs du territoire, lors de la fête des Skira, Hélios est un acropolitain de longue date et aurait déjà une fonction ancestrale : uni à Gè kourotrophos il a engendré les Tritopatores. Certes, l'intervention du Pythien dans la généalogie royale, reconnaît M.V.G., s'est glissée dans une version plus ancienne (p. 23) : le couple Xouthos + Créuse, lié semble-t-il à la formation du territoire et à l'intégration de la Tétrapole (Xouthos) dans le synécisme autour de l'Acropole (Créuse), a précédé le couple Apollon Pythien + Créuse; Ion a certainement été fils de Xouthos (Hérodote) avant d'être celui d'Apollon Pythien (Euripide). Mais cela ne prouve pas que la paternité d'Apollon soit une invention d'Euripide (p. 25). Le culte du Pythien est en effet attesté dans la caverne de l'Acropole dès le VI $\mathrm{s}$. Il se peut donc que, dès cette époque, le mythe d'Ion ait croisé celui d'Apollon Pythien. D’un chapitre à l'autre, qu'il s'agisse de Gè, d'Héphaïstos ou d'Érichthonios, M.V.G. suit le même modèle de démonstration. C'est ainsi que Gè, la terre de l'Attique unifiée (p. 54), est liée à Érechthée dès le VIIe s. ainsi que l'atteste la fête des Genesia, et assume déjà une fonction «kourotrophique » : la figure de Gè kourotrophos, dont le culte est attesté à l'époque romaine sur l'Acropole, remonte à l'époque archaïque et Kourotrophos, la déesse à nom d'épiclèse de l'Acropole, est sans doute la Terre elle-même (p. 57). Que Gè ait été kourotrophos dès l'origine, pourquoi pas ? La plupart des divinités féminines le sont! Mais il est un peu risqué, après la mise au point de St. Georgoudi, citée comme référence, de voir en elle l'énigmatique Kourotrophos de l'Acropole.

L'antériorité des divinités intervenant dans la naissance d'Érichthonios établie, M.V.G. s'applique à démontrer que les figures qui leur sont attribuées sont en rapport avec les grands changements apportés par la politeia de Solon. Il n'est pas question de mettre en doute l'importance du contexte politico-social dans la nouvelle sémantisation de ces représentations, mais les sables sur lesquels nous reconstituons les realia sont si mouvants que la démarche est souvent réversible: si l'histoire peut servir à expliquer la fiction, la fiction permet bien souvent de déchiffrer l'histoire. Un exemple de systématisation difficile : le cas d'Apollon Patroos (p. 3539). Pour M.V.G., Solon ayant intégré le dèmos dans la politeia, un ancêtre fédérateur Apollon Patroos se substitue à Zeus Patroos lié aux Eupatrides et une restructuration du corps civique est placée sous son patronage. Influencés par l'organisation des cités de l'Ionie, les Athéniens remodèlent la partition tripartite de leur corps civique pour adopter le système des quatre tribus dont les héros éponymes deviennent les fils de Ion. La filiation apollinienne de ce dernier fait de l'Attique la plus antique et la plus prestigieuse terre d'Ionie au moment où la cité commence à s'intéresser à cette région. Peut-être! Il y a quelques occurrences pour suggérer une telle interprétation. Mais l'A.P. d'[Aristote], la source fondamentale sur cette question, va à l'encontre de cette hypothèse. Pour son auteur, la partition de la collectivité masculine en groupes spatio-temporels héréditaires à vocation religieuse/civique/politique/militaire est, à Athènes, une constante, depuis la mise en place de la cité des archontes, au lendemain de la disparition de la royauté, jusqu'à son époque, c'est-à-dire le dernier quart du IVe s. La cité, affirme-t-il, n'a eu que deux dispositifs. Le premier - 4 tribus $/ 12$ phratries/48 naucraries - est bien antérieur à Solon. Il est fossilisé par Clisthène qui le réduit à des fonctions civico-religieuses et lui superpose le second dispositif, celui des 10 tribus. Tous ces groupes sont des groupes héréditaires qui se disent dans le langage de la parenté mais qui ne sont pas des groupes de parenté : la parenté réelle, en lignes directe et collatérale, est circonscrite par l'anchisteia. Chaque groupe se veut une confrérie descendant d'un ancêtre commun. L'ancestralité (pour employer le terme de M.V.G.) et son pouvoir fusionnel ne sont donc une invention, ni de la démocratie, ni de Solon. Cette structuration de la collectivité masculine en pseudo-parentés est inhérente, 
en pays grec, à l'organisation en cité où elle assure le passage des sociétés lignagères aux sociétés politiques. La même source attribue à Ion la fondation des quatre tribus, dont certainement la paternité de leurs éponymes. À quel moment Apollon Patroos apparait-il dans le dispositif? Dès l'origine? Lorsque le corps civique a dû intégrer les nouveaux citoyens institués par Solon? La présence du dieu dès le milieu du VIe s. sur l'agora dite de Solon - le nouvel espace de rassemblement du corps civique, le futur meson de la polis -, est certainement en rapport avec le fonctionnement des institutions qui, depuis Solon, intègrent tous les hommes libres. Pour tenir ecclesia ou béliée, les Athéniens se rassemblent tribus par tribus, phratries par phratries. Le culte d'Apollon Patroos assure dans la similitude la cohésion des tribus comme le culte à Zeus Phratrios et Athéna Phratria celle des phratries.

Avec ses quatre «tiroirs », le plan choisi par M. V.G. n'est pas sans provoquer des redites. Il décentre surtout, me semble-t-il, la démonstration par rapport au thème annoncé : pourquoi le fils de la terre, Érichthonios, a-t-il pris la place du fils de la terre, Érechthée ? Cette question réactive un débat sur la maternité de la terre qui a beaucoup divisé en son temps. Est-ce la femme qui imite la terre (J.-P. Vernant) ? Est-ce la terre qui imite la femme (N. Loraux) ? En dépit des apparences, cet échange n'a rien de ludique ! Si, dans le mythe d'Érichthonios, la terre imite la femme, elle n'a, d'après la doxa de l'époque sur la reproduction, aucun rôle dans la procréation d'Érichthonios, elle n'est qu'une mère porteuse. Héphaïstos est le géniteur, la terre se borne à nourrir l'homoncule que le sperme du dieu a déposé en elle. Certes, c'est Gè qui tend l'enfant à Athéna mais c'est Héphaïstos qui, en tant que père géniteur, le donne en adoption à la déesse. Ce n'est pas un hasard si, sur les images, le dieu est toujours en retrait. Il s'efface pour que le père social, en l'occurrence Athéna, puisse soulever l'enfant posé sur le sol et en faire son fils. N.L. constate que, dans le mythe d'Érichthonios, la terre évince la femme de la naissance du premier citoyen. L'argument est, je crois, réversible: parce qu'elle imite la femme la terre est éliminée de la procréation du premier citoyen. Pour le dire autrement, la terre nourrit Érichthonios et ses descendants, elle ne les engendre pas. La possession d'un lot de la terre civique ne leur donne pas le statut de citoyen, mais leur statut de citoyen leur assure d'être nourri par la terre civique. C'est alors qu'apparait en pleine lumière ce qui distingue Érichthonios d'Érechthée. Érechthée est un fils de la terre qui apparemment l'a enfanté seule. Érechthée n'a pas de père : son statut dépend de sa mère. Le groupe de descendance d'Érechthée ne comprend que des détenteurs du sol. À mon avis, ce n'est pas avec un hypothétique processus de développement de la petite propriété paysanne consécutif à la réforme de Solon qu'il faut mettre en rapport le passage d'Érechthée à Érichthonios mais avec le refus de Solon de procéder à un nouveau partage de «la grasse terre de la patrie». À partir du moment où l'archonte a fondé la citoyenneté sur la liberté et non sur la détention d'un lot de la terre civique, la réorganisation de la tradition homérique s'impose : les Athéniens doivent se raconter et raconter aux autres Grecs que le premier citoyen n'avait pas été engendré par la terre, mais seulement nourri par elle. La figure du géniteur est essentielle. Pourquoi avoir dévolu ce rôle à Héphaïstos ? Parce qu'il est le dieu des artisans qui le célèbrent lors des Chalkeia et que l'artisanat est en plein développement sous la tyrannie ? Peut-être ! Mais, en pays grec, la tradition religieuse est poésie. Le dieu de la flamme jaillissante entretient, je crois, avec la déesse à l'olivier des relations que seul le poète inspiré pouvait, dans le langage qui est le sien, faire découvrir à son auditoire.

Quelle conclusion tirer d'aussi longues remarques, sinon que l'ouvrage de M.V.G., est, en dépit d'une construction parfois un peu déconcertante, un livre qui provoque la réflexion.

Claudine Leduc

(Université de Toulouse - Le Mirail) 